\title{
Rolipram potentiates bevacizumab-induced cell death in human glioblastoma stem-like cells
}

\author{
Sara Ramezani ${ }^{1,2}$, Nasim Vousooghi ${ }^{1,3}$, Fatemeh Ramezani Kapourchali ${ }^{4}$, Mahmoudreza Hadjighasem ${ }^{1,5}$, Parisa
} Hayat $^{6}$, Naser Amini ${ }^{6}$, Mohammad Taghi Joghataei ${ }^{1,6,7^{*}}$

\footnotetext{
${ }^{1}$ Department of Neuroscience, School of Advanced Technologies in Medicine, Tehran University of Medical Sciences, Tehran, Iran

${ }^{2}$ Neuroscience Research Center, School of Medicine, Guilan University of Medical Sciences, Rasht, Iran

${ }^{3}$ Iranian National Center for Addiction Studies (INCAS), Tehran University of Medical Sciences, Tehran, Iran

${ }^{4}$ Department of Human Nutritional Sciences, University of Manitoba, Winnipeg, MB, Canada

${ }^{5}$ Brain and Spinal Cord Injury Research Center, Tehran University of Medical Sciences, Tehran, Iran

${ }^{6}$ Cellular and Molecular Research Center, Iran University of Medical Sciences, Tehran, Iran

${ }^{7}$ Neuroscience Department, School of Advanced Technologies in Medicine, Iran University of Medical Sciences,

Tehran, Iran

*Corresponding author; Tel: (+98)121114191; Tel. \& Fax: (+98) 21867047 20;

Email: mt.joghataei@yahoo.com
}

(C) 2017. This manuscript version is made available under the Elsevier user license http://www.elsevier.com/open-access/userlicense/1.0/ 


\begin{abstract}
Aims: Glioblastoma cancer stem-like cells (GCSCs) promote themselves proliferation by secreting the vascular endothelial growth factor $\mathrm{A}\left(\mathrm{VEGF}_{\mathrm{A}}\right)$ in an autocrine manner, positively regulated by phosphodiesterase IV (PDE4). In the current study, we investigated the putative cytotoxic effect of bevacizumab, a $\mathrm{VEGF}_{\mathrm{A}}$ blocker, alone and in combination with a specific inhibitor of PDE4 called rolipram on GCSCs isolated from human surgical tumor specimen with a focus on PI3K/AKT pathway.

Main Methods: CD133+/CD15+ GCSCs were characterized by flow cytometry and expanded in a serum-free primary culture system. The cell survival, apoptosis, and protein expression values were measured using MTT assay, TUNEL staining and western blot, successively. Intracellular cAMP and free secreted $\mathrm{VEGF}_{\mathrm{A}}$ levels were assessed by cAMP enzyme immunoassay and ELISA, respectively.

Key findings: Bevacizumab suppressed GCSCs survival with $\mathrm{IC}_{50} \sim 6.5 \mu \mathrm{g} / \mathrm{ml}$ and enhanced the levels of apoptosis, p53 and cleaved-caspase 3 along with a decrease in free $\mathrm{VEGF}_{\mathrm{A}}$ levels and ERKs activation. However, there was no significant modulation of AKT phosphorylation on serine 473, the intracellular PDE4A, VEGF $_{\mathrm{A}}$ and cAMP levels. More cytotoxicity in co-treated cells coupled with a more substantial decline in the free $\mathrm{VEGF}_{\mathrm{A}}$ levels and a greater increase in the quantities of p53 and cleaved-caspase 3 compared to those treated with bevacizumab alone. Cotreatment reduced phospho-AKT, endogenous $\mathrm{VEGF}_{\mathrm{A}}$ and PDE4A values but elevated cAMP levels.

Significance: This study highlighted a booster cytotoxic effect of combined rolipram and bevacizumab treatment on the GCSCs primary culture, suggesting that this approach is warranted in treatment of GBMs overexpressing VEGF $_{\mathrm{A}}$ and PDE4A.
\end{abstract}

Key words: Glioblastoma multiforme, Co-treatment, VEGF $_{\mathrm{A}}$, PDE4A, Cyclic AMP, p53, AKT 


\section{Introduction}

Glioblastoma multiforme (GBM) is the most common malignant primary brain tumor in adults [1]. Despite standard therapeutic interventions, the patients succumb to death within 12-15 months after diagnosis [2,3]. The evidence supports the existence of a sub-population of GBM cells known as glioblastoma cancer stem-like cells (GCSCs) having the potential of tumorigenesis [4-6] and chemo-resistance and radio-resistance [7,8]. These cells are characterized by some cell surface markers including CD133 and CD15[9,10]. It is stated that CD133+/CD15+ GCSCs are capable of generating the sphere-like colonies in vitro $[11,6,10]$. Furthermore, overexpression of phosphodiesterase IVA (PDE4A) and vascular endothelial growth factor (VEGF) in human GBM tissues have been identified, associated with unfavorable clinical outcome [12] and intracranial tumor growth[13].

Indeed, an essential VEGF isotype to progress angiogenesis is $\mathrm{VEGF}_{\mathrm{A}}$, mostly secreted from GCSCs. $\mathrm{VEGF}_{\mathrm{A}}$-dependent growth promoting signal is mainly transmitted via VEGF receptor 2 (VEGFR2) [14-16]. GCSCs promote angiogenesis through the secretion of $\mathrm{VEGF}_{\mathrm{A}}$ in a paracrine manner on the endothelial cells [17,18]. In addition, GCSCs boosts the survival and proliferation themselves via VEGF $_{\mathrm{A}}$-VEGFR2 interplay under a positive autocrine loop of $\mathrm{VEGF}_{\mathrm{A}}$ [19-21]. Recently, a research defined the stimulatory function of some PDE4 isoforms in upregulating of $\mathrm{VEGF}_{\mathrm{A}}$ expression [22]. Hence, it is of interest to clarify an ambiguity based on whether the combination of $\mathrm{VEGF}_{\mathrm{A}}$ blocker with the PDE4A inhibitor can confer a pronounced effectiveness on cell growth inhibition in GCSCs by modulating $\mathrm{VEGF}_{\mathrm{A}}$ autocrine loop. In last years, bevacizumab (Avastin), a recombinant humanized monoclonal antibody against $\mathrm{VEGF}_{\mathrm{A}}$ has been developed to inhibit the angiogenesis in recurrent GBM patients [23]. However, it remains to be well elucidated wherever bevacizumab might suppress the GCSCs survival and proliferation. In addition, the mechanism(s) of putative cytotoxicity of bevacizumab is not much known. There is controversial evidence about the direct effect of bevacizumab on proliferation and migration of several cancer cell lines in vitro [24-27]. According to prior observations, bevacizumab paradoxically stimulates cell proliferation in vitro [25] and its continuous administration leads to tumor invasion in patients [28]. It is believed that tumor recurrence after bevacizumab application is related to compensatory activation (phosphorylation) of growth signal

transducers such as protein kinase B (AKT) and extracellular signal-regulated kinase (ERK) belonging to serine/threonine kinases family, probably triggered by other growth factors except for $\mathrm{VEGF}_{\mathrm{A}}[25]$. On the other hand, some reports postulate that rolipram, an inhibitor of PDE4, mediates cell cycle arrest through multiple pathways [29,30] which one of them is AKT inactivation[31]. 
Based on above descriptions, we hypothesized that combining the inhibitors of PDE4A and $\mathrm{VEGF}_{\mathrm{A}}$ may be able to negatively influence proliferation of GCSCs in primary culture through regulating either $\mathrm{VEGF}_{\mathrm{A}}$ autocrine loop or the relevant compensatory growth signals in the absence of $\mathrm{VEGF}_{\mathrm{A}}$. Notably, bevacizumab therapy for GBM patients in a standard therapeutic dosage not only leads to an unstable clinical utility that is presumably related to antiangiogenesis activity of this medicine but also it ultimately faces to the failure often after a few month of treatment onset [28]. Besides, high-dose bevacizumab may be more efficacious [32] however it may be an expensive treatment causing more severe side effects [33]. Hence, we have thought that if the our suggested co-treatment approach can potentiate the cytotoxicity induction and $\mathrm{VEGF}_{\mathrm{A}}$ autocrine loop blockage in GCSCs versus single treatments, the addition of rolipram to low-dose bevacizumab can be potentially considered as a substitute antiangiogenesis and anticancer therapeutic approach to achieve a dramatic constant improvement without tumor refractoriness.

In the current study, we investigated the effect of bevacizumab, a VEGF $\mathrm{Al}_{\mathrm{A}}$ blocker, alone and in combination with a specific inhibitor of PDE4A called rolipram on the GCSCs survival with focused on Phosphoinositide 3-kinase (PI3K) /AKT signal transduction pathway, a key mediator of cell survival, proliferation and chemo-resistance [34,35]. Here, the impact of bevacizumab alone on ERKs activity in GCSCs has also been examined. The endogenous and secreted levels of $\mathrm{VEGF}_{\mathrm{A}}$ were evaluated to determine the effect of drugs on $\mathrm{VEGF}_{\mathrm{A}}$ autocrine loop in GCSCs. The intracellular levels of cAMP in GCSCs treated with bevacizumab alone or accompanied to rolipram were also assessed. Moreover, the role of tumor suppressor transcription factor called p53 in probable cell death was studied in both conditions of the single drug and co-treatment.

\section{Materials and Methods}

\section{Tumor Sampling}

Tumor specimens belonging to three newly diagnosed GBM patients with no prior exposure to radiation or chemotherapy were surgically resected and conveyed to our laboratory based on informed consent written of patients. The protocol was approved by the ethical committee of Iran University of Medical Sciences. The samples were maintained at proper conditions and were labeled as T1, T2 and T3.

\section{Primary culture of GCSCs}

Central zone of samples were freshly subjected, washed with phosphate-buffered saline (PBS; SigmaAldrich, France), minced and enzymatically dissociated via Trypsin, 0.25\% (1x) with EDTA (Gibco; Life 
Technologies, USA) and deoxyribonuclease I (DNase I; Sigma-Aldrich, France). And equal volume of soybean (Invitrogen, USA) was used to stop the trypsin reaction. After centrifuging, the pellet was added RBC lysis buffer (Invitrogen, USA) to remove the red blood cells. Afterward, the suspension was centrifuged and then the pellet was added Dulbecco's Modified Eagle Medium/nutrient mixture F-12 (DMEM/F-12; Gibco Life Technologies, USA). The suspension was filtered through a 70-micron cell strainer (Singapore،SPL). Single cells were plated at density of $5 \times 10^{4}$ cells $/ \mathrm{ml}$ in appropriate cell culture flasks consisting of serum-free medium supplemented with B27 (5X) (Invitrogen, USA) at a 5:1 ratio, $20 \mathrm{ng} / \mathrm{ml}$ human Epidermal Growth Factor (EGF; Invitrogen, USA), 20ng/ml basic Fibroblast Growth Factor (b-FGF; Invitrogen, USA), $2 \mu \mathrm{g} / \mathrm{ml}$ heparin (Invitrogen, USA) and $1 \%$ antibiotic/antimycotic (Invitrogen, USA). Then the cultures were incubated at $37^{\circ} \mathrm{C}$ in a humidified chamber with $5 \% \mathrm{CO}_{2}$. The GCSCs-derived spheres were replated when the average size of them became 200 microns in diameter within one week. The Spheres at passage five were used for the subsequent experiments.

\section{Quantitative Real-time polymerase chain reaction $(q P C R)$}

Total RNA was isolated from central and peripheral parts of human tumor samples belonging to three newly diagnosed GBM patients using RNeasy Tissue Mini Kit (Qiagen, Germany). Complementary DNA (cDNA) was synthesized using Quanti Tect Reverse Transcription Kit (Qiagen, Germany). The quantification of gene expression was carried out using Power SYBR_ Green PCR Master Mix (Life Technologies, Grand Island, NY, USA) on StepOnePlus Real-Time PCR System (Applied Biosystems, Foster City, CA, USA). The oligonucleotide primers of beta-actin (housekeeping gene) and $\mathrm{VEGF}_{\mathrm{A}}$ (target gene) were provided by Qiagen company primer bank. Obtained data of $\mathrm{VEGF}_{\mathrm{A}}$ mRNA was normalized using beta-actin. Relative expression of $\mathrm{VEGF}_{\mathrm{A}}$ was quantified by Relative Expression Software Tool (REST)-XL version 2 [36]. In order to calculate the significance of results, pair-wise fixed reallocation randomization test was applied. All experiments were done in duplicate.

\section{Flow cytometry Analysis}

In order to characterize the GCSCs, CD133 and CD15 as positive markers and CD34 and CD45 as negative markers were detected using flow cytometry. To do that, the cells at $5^{\text {th }}$ passages were harvested and centrifuged at $1200 \mathrm{rpm}$ for $5 \mathrm{~min}$, fixed with 4\%paraformaldehyde, permeabilized using 0.25\% Triton-X100. After washing with PBS twice, the cells resuspended in PBS and then labeled with primary antibodies such as CD133, CD15, CD45 and CD34 (Abcam, USA) for $60 \mathrm{~min}$ at $4^{\circ} \mathrm{C}$ in dark afterward incubated with secondary goat anti-rabbit antibodies conjugated with phycoerythrin (PE) or FITC (Abcam, USA) for $60 \mathrm{~min}$ at room temperature in dark. The cells were 
stained with FITC or PE-conjugated rabbit monoclonal IgG1 antibodies (Abcam, USA) as isotype controls. The cells were monitored using BD FACScalibur flow cytometer device (BD Biosciences, USA).

\section{Treatments with the Drugs}

The GCSCs isolated from T2 at $5^{\text {th }}$ passages having a purity of more than $95 \%$ based on the assessment of CD133 and CD15 expressions were harvested and seeded in 24-well plates containing DMEM-F12 at a density of $2.5 \times 10^{4}$ cells /well, overnight. The cells were treated with various concentrations $(1,3,10,30,100 \mu \mathrm{g} / \mathrm{ml})$ of bevacizumab (Roche, Switzerland) for $48 \mathrm{~h}$. All treatments were done in quadruplicate. After finding of half maximal inhibitory concentration $\left(\mathrm{IC}_{50}\right)$ of bevacizumab, the cells incubated with bevacizumab at $\mathrm{IC}_{50}$ in the absence or presence of rolipram (103 $\mu$ M). Human Immunoglobulin G1 (IgG1; Sigma-Aldrich, France) at the same volume was used instead bevacizumab as control.

\section{MTT Assay}

At days $0,2,4,6$ and 8 of primary culture, as well as at the end of treatment period, the cells were incubated with $5 \mathrm{mg} / \mathrm{ml}$ mehtylthiotetrazole (MTT) solution (Sigma-Aldrich, France) for $4 \mathrm{~h}$. After centrifuging, the medium was carefully discarded and Dimethyl sulfoxide (DMSO; Sigma-Aldrich, France) was added to samples. Optical density (OD) was read at $570 \mathrm{~nm}$ by microplate reader (BioTek, USA). The average of quadruplicate absorbance readings values for each experimental condition was measured, separately.

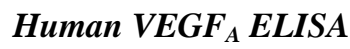

Human VEGF DuoSet ELISA kit (R\&D, UK) was utilized to measure secreted VEGF $_{\mathrm{A}}$ levels in culture medium at $48 \mathrm{~h}$ after treatments, according to the manufacturer's instructions. The absorbance at $450 \mathrm{~nm}$ was evaluated by a microplate reader (Bio Teck, USA), and soluble $\mathrm{VEGF}_{\mathrm{A}}$ concentrations were calculated based on a standard curve.

\section{Cyclic AMP measurement}

The intracellular levels of cyclic AMP were assessed by Correlate-EIA ${ }^{\mathrm{TM}}$ Cyclic AMP Enzyme Immunoassay Kit (Assay designs, Inc. USA) according to the manufacturer's instructions. Briefly, At $48 \mathrm{~h}$ after treatments, T2-derived GCSCs collected, lysed in $0.1 \mathrm{M} \mathrm{HCl}$ solution and removed by centrifugation at $>600 \times g$ for $10 \mathrm{~min}$. Assay procedure running was initiated when lysates were resuspended in cAMP assay buffer (Assay Designs) At termination, all reactions were laid off. Immediately, absorbance at $405 \mathrm{~nm}$ was read by a microplate reader (Bio Teck, USA), and cAMP concentrations were calculated based on a standard curve. 


\section{TUNEL Staining}

Terminal deoxynucleotidyl transferase dUTP nick end labeling (TUNEL) staining was carried out according to manufacturer's instructions (In Situ Cell Death Detection Kit, Fluorescein - Roche applied science, Switzerland). Briefly, the cells were fixed in fresh $4 \%$ paraformaldehyde at room temperature for 60 min and then rinsed with PBS twice and incubated with $0.1 \%$ Triton X-100 solution (Sigma-Aldrich, France) at $8^{\circ} \mathrm{C}$ for 2 min. After washing, the cells were incubated with 50 $\mu$ l TUNEL reaction mixture at $37^{\circ \mathrm{C}}$ in a humidified chamber for 60 min. The cells were counterstained with propidium iodide (PI; Sigma-Aldrich, France) and visualized using Fluorescence inverted microscope (Olympus IX71). Apoptosis value was semi-quantified by calculating TUNELpositive cells number of each field relative to PI-positive cells number (total cells) of the same field using Image $\mathbf{J}$ software.

\section{Western Blot Analysis}

All experimental samples were lysed using the lysis buffer containing RIPA (Cell Signaling Technology, Netherlands) supplemented with protease inhibitor and phosphatase inhibitor cocktails (Roche, Switzerland) on ice for $30 \mathrm{~min}$. Afterward, the mixture was centrifuged at $13000 \mathrm{~g}$ for $20 \mathrm{~min}$ at $4^{\circ} \mathrm{C}$. Bradford method was used to determine Protein concentration [37]. Equal values of proteins $(80 \mu \mathrm{g})$ were loaded on sodium dodecyl sulfate (SDS) polyacrylamide gel and separated in a size manner by electrophoresis. Proteins were transferred to nitrocellulose membranes (Amersham Biosciences, USA). The membranes were blocked with 5\% skim milk in Trisbuffered saline (TBS; pH 7.4). Membranes incubated with primary antibodies for PDE4A, VEGFR1/2, AKT1, phospho-AKT1 (Ser473), p53, cleaved caspase3 (aspartate-specific cysteine protease3) and $\beta$-actin (Abcam, USA), p-ERK1/2 (Thr202/Tyr204) or phospho-p44/42 mitogen-activated protein kinase (MAPK), ERK1/2 (p44/42 MAPK) (Cell Signaling, France) and $\operatorname{VEGF}_{\mathrm{A}}\left(\right.$ Santa Cruz, USA) overnight at $4^{\circ} \mathrm{C}$. After washing, membranes incubated with goat anti-rabbit secondary antibody conjugated with horseradish peroxidase (HRP). All antibodies were diluted according to manufacturer's instructions. Immunoreactive bands were visualized using an enhanced chemiluminescence (ECL) detection system (Amersham Biosciences, USA). X-ray films were scanned, and then the relative protein levels were semi-quantified by densitometric analysis using Total lab software (Bio Step). Beta-actin was tested as the internal control.

\section{Statistical analysis}


All data were analyzed using SPSS software (version 22) and the results were presented as mean \pm standard error (SE). The differences in values of cell viability, apoptosis, intracellular cAMP, protein expressions and extracellular soluble free $\mathrm{VEGF}_{\mathrm{A}}$ among treatment groups were examined using the one-way analysis of variance (ANOVA) and post hoc test as pairwise comparisons. Independent sample t-test was applied to analyze differences in expression values of PDE4A between central and peripheral tissues of tumors as well as to compare the relative values of p-ERKs (Thr202/Tyr204) expression between bevacizumab-treated cells and those exposed to human IgG1 as control. Difference were considered statistically significant at $* P<0.05, * * P<0.01$, *** $p<0.001$. Nonlinear regression analysis by Prism graph pad 6 software was utilized to determine IC50 of bevacizumab.

\section{Results}

\section{Characterization of patients and GCSCs purity in primary cultures}

Basic characteristics of patients and their tumors were illustrated in table1. Flow cytometry data elucidated that cancer cells isolated from $\mathrm{T} 1$ and $\mathrm{T} 3$ at $5^{\text {th }}$ passage of primary culture slightly express positive markers of GCSCs, indicating little purity of GCSCs in primary cultures of T1 and T3. However, about 97\% of T2- extracted cells represented CD133 and CD15 markers, signifying very high purity of GCSCs in T2 primary culture. As shown in Fig. 1A, the expression of CD133 or CD15 or both of them were detected for almost $42.18 \%, 13.34 \%$ and $40.87 \%$ of T2-isolated cell population, respectively. Approximately, 8\% and 7\% of the T2-isolated cells expressed CD45 and CD34, successively. It holds out the negligible presence of hematopoietic stem cell subpopulation within primary culture of T2-extracted cells.

\section{Colony forming ability and growth rate of tumor-isolated cells in primary cultures}

The cells from all samples generated neurosphere-like colonies, termed GBM spheres within $48 \mathrm{~h}$ of primary culture. Interestingly, T2-derived GBM spheres unlike spheres formed from T1 or T3 remained nonadherent and intensively continued to proliferate until seven days after plating, when they were readied to subculture (Fig. 1B). Likewise, the cells from T2 sample demonstrated the more substantial increase in clonogenic and proliferative capacities relative to the cells from other samples; especially from day 4 to day eight after plating (Fig. 1C). Hereby, T2-isolated GCSCs qualified to do subsequent experiments regarding the investigation of cytotoxic effect of therapeutic agents.

The expressions of $\mathrm{VEGF}_{A} \mathrm{mRNA}$ and PDE4A and VEGFR1/2 proteins in tumor samples 
According to obtained results, the mean of relative PDE4A expression values in central tissue of samples $(1.77 \pm 0.23)$ was significantly higher than the mean of those in peripheral zone $(0.61 \pm 0.1)$ (Fig. $2 \mathrm{~A})$. The relative expression of $\mathrm{VEGF}_{\mathrm{A}}$ mRNA was about 3.5 fold more in central zone than peripheral tissue (Fig. 2B). The cells isolated from central zone of all tumor tissues exhibited positive immunoblotting for VEGFR2. However, pronounced expression of VEGFR1 only appeared in T1 cells culture (Fig. 2C).

Bevacizumab induces cytotoxicity, sequestrates free $\mathrm{VEGF}_{A}$ and lessens ERKs activation; the co-treatment with rolipram intensifies secreted $\mathrm{VEGF}_{A}$ decay in GCSCs culture medium

Incubation of GCSC with bevacizumab reduced the percent of viable cells, in a dose-dependent manner. GCSCs were sensitive to bevacizumab, with IC50 value of approximately $6.5 \mu \mathrm{g} / \mathrm{ml}$ (95\% Confidence Interval [CI] $=4.93-8.67$ ) (Fig. 3A). Interestingly, bevacizumab led to a significant decrease in the levels of ERKs phosphorylation about two-fold of control (Fig. 3B). The free soluble $\mathrm{VEGF}_{\mathrm{A}}$ levels in culture medium from untreated GCSCs was quantified approximately $451 \pm 27.2$ (pg/ml/million cells). Predictably, bevacizumab after $48 \mathrm{~h}$

markedly decreased free soluble $\mathrm{VEGF}_{\mathrm{A}}$ levels in cell culture supernatant compared with rolipram and control. A significant reduction of free $\mathrm{VEGF}_{\mathrm{A}}$ levels in rolipram-treated culture medium also emerged as compared to control. The co-treatment strategy depleted the levels of free $\mathrm{VEGF}_{\mathrm{A}}$ more than rolipram or bevacizumab alone (Fig. 3C).

Co-treatment with rolipram and bevacizumab augments cytotoxic activity exerted by each treatment alone and elevates intracellular cAMP levels similar to Rolipram

Rolipram alone or in combination with bevacizumab could elevate by 4 fold intracellular cAMP levels relative to control. No significant change in the cAMP levels of bevacizumab-treated GCSCs was observed (Fig. 4A). Rolipram or bevacizumab resulted in cell viability reduction compared to control. Interestingly, co-treated cells with bevacizumab plus rolipram indicated a significant decrease in the cell viability in comparison with each treatment alone and control (Fig. 4B). It was revealed that both rolipram and bevacizumab considerably induced cell apoptosis, individually. The co-treatment of the GCSCs with rolipram plus bevacizumab extremely impeded the apoptosis rate as compared to those treated with each therapeutic agent alone (Fig. 4C).

The levels of PDE4A was attenuated in co-treated GCSCs and rolipram-treated ones, similarly but more than those treated bevacizumab

Rolipram alone and in combination with bevacizumab individually brought about the significant decrease in the intracellular levels of PDE4A expression versus both control and bevacizumab alone. However, the GCSCs 
treated with bevacizumab alone did not statistically show any significant reduction of PDE4A levels in comparison to control. Interestingly, downregulation of PDE4A induced by co-treatment did not statistically differ from that produced by rolipram alone (Fig. 5).

\section{Rolipram alone but not bevacizumab similar to co-treatment approach abolishes AKT phosphorylation on Serine 473 and endogenous VEGFA levels in GCSCs}

The calculation of expression ratio of phospho-AKT1 (Ser473)/total AKT1 from three independent experiments clarified that the mean of relative levels of p-AKT (ser473) was not statistically altered in GCSCs exposed to bevacizumab compared to control. Surprisingly, the incubation of GCSCs with bevacizumab had no effect on the levels of endogenous $\mathrm{VEGF}_{\mathrm{A}}$ expression which remained similar to control cells. However, the relative levels of phospho-AKT (ser473) and VEGF $_{\mathrm{A}}$ were significantly depleted in co-treated GCSCs with rolipram plus bevacizumab and those treated with rolipram alone (Fig. 5).

\section{Co-treatment with rolipram and bevacizumab potentiates p53 tumor suppressor in GCSCs}

Bevacizumab and rolipram individually led to the significant increase in the relative values of p53 and cleaved-caspase 3 expressions. Notably, co-treatment method profoundly augmented the amount of p53 upregulation as compared to each treatment alone. Furthermore, the overactivation of caspase 3 caused by each treatment alone was strongly amplified when bevacizumab and rolipram added to culture medium, simultaneously (Fig. 5)

\section{Discussion}

Mechanistically, the sequel of bevacizumab application in cultures containing GCSCs with no presence of endothelial cells remains to be well elucidated. In the current study, we could find that bevacizumab directly exerted antiproliferative and proapoptotic properties in GCSCs. We also discovered that bevacizumab imposed its cytotoxic effect through inhibiting ERKs signaling pathway. It was revealed that p53 upregulation by bevacizumab took part in triggering the proapoptotic molecular cascades. This study eliminated that the presence of rolipram concomitant with bevacizumab conferred an additional advantage to the antitumor efficacy of bevacizumab in GCSCs culture.

Generally, there is little information about the direct effect of bevacizumab on human cancer cells because in-vitro studies of bevacizumab have been predominantly focused on endothelial cells, so far. However, the investigations in this field demonstrated diverse contradictory findings based on tumor cell type-specific responses into bevacizumab [24-27]. According to ongoing study, a previous effort suggested the cytotoxic activity of bevacizumab on adenocarcinoma cell line [26]. Wang and colleagues suggested that bevacizumab-induced apoptosis 
of adenocarcinoma cells was linked to endoplasmic reticulum stress. Nevertheless, the present findings concerning the cell viability after bevacizumab application did not corroborate some prior observations [25]. This discrepancy may result from differences in methodological perspective of studies. Undoubtedly, heterogeneity in GBM cancer cells and variety of bevacizumab concentrations applied in different studies could interfere in this discordance of the results. In the study of Simon and colleagues, the cell lines expressing VEGFR1 but not VEGFR2 indicated a significant proliferative response to bevacizumab along with upregulation of placental growth factor (PIGF) and VEGFR1, while T2-isolated GCSCs expressing VEGFR2 in our study did not represent VEGFR1. Likewise, Simon and colleagues showed that bevacizumab at $1 \mathrm{mg} / \mathrm{ml}$ depleted free $\mathrm{VEGF}_{\mathrm{A}}$ levels by 10 fold relative to control in various GBM cell lines, while in our study, bevacizumab at $6.5 \mu \mathrm{g} / \mathrm{ml}$ diminished free $\mathrm{VEGF}_{\mathrm{A}}$ levels by 3 fold compared to control. Thus, it seems that high concentrations of bevacizumab are exactly in favor of more free $\mathrm{VEGF}_{\mathrm{A}}$ sequestration and subsequently strong excitation of pro-proliferative signals, presumably through other growth factors. Therefore, it thought that in a monotherapy approach based on the blockade of $\mathrm{VEGF}_{\mathrm{A}}$ autocrine loop, the efficient suppression of cancer cells proliferation relays on the therapeutic agent dosage adjusted according to free $\mathrm{VEGF}_{\mathrm{A}}$ levels with no activation of compensatory growth pathway.

We also clarified that bevacizumab- induced cytotoxic effect was associated with a meaningful decline in the activation of ERKs but not AKT. The lack of conspicuous change in AKT phosphorylation levels by bevacizumab may stem from the intracellular homeostatic process mediated by unknown cytokines to maintain cell growth-promoting signals. Albeit, it was cleared that bevacizumab brought about a considerable decay of free $\mathrm{VEGF}_{\mathrm{A}}$ levels within culture medium, it could not produce any modulation of intracellular $\mathrm{VEGF}_{\mathrm{A}}$ values. Functionally, AKT kinase stands on the top of signal transduction cascades triggering $\mathrm{VEGF}_{\mathrm{A}}$ gene transcription and translation [38]. Accordingly, it is conceivable that the levels of intracellular $\mathrm{VEGF}_{\mathrm{A}}$ along with AKT phosphorylation status were constantly retained within the cell through a resistance mechanism against free $\mathrm{VEGF}_{\mathrm{A}}$ blockage. Furthermore, it is possible that unchanged levels of intracellular PDE4A and cAMP connect to the preservation of intracellular $\mathrm{VEGF}_{\mathrm{A}}$ and $\mathrm{AKT}$ phosphorylation levels in bevacizumab-treated cultures.

Rolipram weakened the amount of cell viability consistent with the enhancement of intracellular cAMP levels and the decrease in the levels of AKT phosphorylation, intracellular $\mathrm{VEGF}_{\mathrm{A}}$ and PDE4A, as well as soluble free $\mathrm{VEGF}_{\mathrm{A}}$ in medium culture. Surprisingly, rolipram when simultaneously applied to bevacizumab could yield a prominent reduction of AKT phosphorylation, intracellular PDE4A and $\mathrm{VEGF}_{\mathrm{A}}$ levels and a dramatic elevation of 
cAMP levels similar to rolipram alone. These findings signify that rolipram is exclusively responsible for these alterations in co-treated cultures. It is guessed that in cultures treated with rolipram alone, PDE4A reduction-induced elevation of intracellular cAMP levels might promote cytotoxicity through inhibiting AKT which transmits the regulatory signals of $\mathrm{VEGF}_{\mathrm{A}}$ expression. Moreover, rolipram-induced VEGFA autocrine loop blockade surely participates in the suppression of cell growth and survival promoting processes.

It is worth noting that remarkable cell death arises from co-treatment approach was in concurrent with a visible enhancement in intracellular cAMP levels and a more marked depletion of free secreted $\mathrm{VEGF}_{\mathrm{A}}$, intracellular PDE4 and AKT phosphorylation levels in comparison with bevacizumab treatment. A prior study manifested that cAMP elevators could activate protein tyrosine phosphatases (PTPs) [39]. Hence, a possibility to explain the outstanding cytotoxicity despite a more potent drop of free $\mathrm{VEGF}_{\mathrm{A}}$ levels in co-treated cells versus bevacizumab-treated ones is that the addition of rolipram to bevacizumab might suppress either the primary step of growth signal transduction that is receptor tyrosine kinases (RTKs) phosphorylation, presumably via stimulating PTPs activity or downstream effectors of RTKs like AKT [40,30], as observed in this study. In addition, it cannot be excluded that the more loss of free secreted $\mathrm{VEGF}_{\mathrm{A}}$ levels may involve in the more abrogation of cell proliferation in co-treated culture versus bevacizumab-treated ones. Likewise, co-treatment could produce a more pronounced decrease in secreted free $\mathrm{VEGF}_{\mathrm{A}}$ levels compared with rolipram alone. Herein, it is suggested that in a combination approach, the treatments collaboratively barricade $\mathrm{VEGF}_{\mathrm{A}}$ autocrine loop in GCSCs, leading to an augmentative cytotoxicity induction.

On the basis of these results, it is thought that in a co-treatment system, the presence of rolipram can permit to reduce the therapeutic dose of bevacizumab used in the clinic because this combination system represents both intensive antitumor and antiangiogenesis potentials through inhibiting AKT pathway and $\mathrm{VEGF}_{\mathrm{A}}$ autocrine loop in GCSCs, possessing clinical significance.

Bevacizumab resulted in the apoptosis induction in parallel with the upregulation of p53 and cleavedcaspase 3 levels, as well as the suppression of ERKs signaling pathway. On the other hand, in this assay according to prior studies, rolipram alone induced the cell apoptosis and boosted the levels of p53 [41,42] and fragmentedcaspase3 along with AKT inactivation. Fundamentally, growth signal transducers including ERK and AKT stimulate E3 ubiquitin ligase activity of murine double minute 2 (MDM2), leading to p53 degradation [43,44]. It is thought that bevacizumab and rolipram independently inhibit ERK and AKT activity, respectively and consequently 
abrogate MDM2-mediated p53 degradation, leading to the activation of proapoptotic processes. In fact, P53 promote the intrinsic apoptotic pathway via a transcription dependent/independent mechanism [45]. Likewise, p53 actually implicated in the transition of death receptors signals promoting the extrinsic apoptosis via a transcription-dependent mechanism [46]. Previous studies demonstrated that an intrinsic apoptotic mechanism conducts rolipram-induced apoptosis [41,30]. However, there is no evidence based on the proapoptotic action of bevacizumab through the intrinsic pathway. Furthermore, a previous study displayed that an increase in post-treatment serum levels of tumor necrosis factor (TNF)-related apoptosis-inducing ligand (TRAIL) were correlated with higher median survival in patients with metastatic colon cancer following bevacizumab therapy [47]. It denotes that bevacizumab presumably contributes the apoptosis induction via the extrinsic pathway in which particular death receptors such as agonistic receptors of TRAIL are engaged to trigger the caspases cascade [45]. Thus, TLAIR-initiated apoptotic pathway might be exposed to discuss as a feasible alternative mediator of apoptosis due to bevacizumab treatment in our study that is recommended to explore in the next studies. It was disclosed that co-treatment approach additively reinforced the rate of cell apoptosis induction linking to the amplification of p53 and cleved-caspase3 upregulations as compared to single treatments. It should be noted that alleged extrinsic and mitochondrial intrinsic apoptotic pathways converged in the final steps of apoptotic cascades that one of them is caspase3 activation [46]. As an explanation, it is conceivable that the separated potency of each treatment alone for enhancing the levels of p53 and triggering the p53-mediated proapoptotic cascades is integrated together in a concurrent system of drugs application, and argumentatively intensifies the activation of caspase3, leading to a major apoptosis induction.

Altogether, these results were obtained from CD133+/CD15+ cancer cells expressing VEGFR2 but not VEGFR1 and high levels of $\mathrm{VEGF}_{\mathrm{A}}$ and PDE4A. Thereby, it is necessary that the mentioned biomarkers in future efforts are considered to effectively translate the laboratory findings of this new combination therapy into medical practice. Indeed, further investigations can prove the significance of biomarkers such as PDE4A, VEGF ${ }_{\mathrm{A}}$, and VEGFR1/2 to identify the patients that are most likely to profit from this novel combination strategy. It should be mentioned that our study was conducted only on glioblastoma cells and not the normal cells. This may be a limitation of this study. However, we are planning to evaluate the effect of our suggested co-treatment on normal cells in the future. Nevertheless, it has been shown previously that neither of these drugs has identifiable toxic effects on normal cells such as retinal or spiral ganglion neurons and normal circulating hematopoietic cells [48-50].

\section{Conclusion}


In overall, our experiments suggest the possibility that in GCSCs cultures, cell growth suppression and apoptosis induction by bevacizumab may be mediated through p53 upregulation, ERKs deactivation and free secreted VEGFA block. It is also revealed that rolipram-induced cell growth inhibition and apoptotic cell death are associated with a decrease in the levels of PDE4A, phospho-AKT (ser473) as well as blockade of VEGF $_{\mathrm{A}}$ autocrine loop and p53 upregulation in GCSCs. Notably, in spite of the more cytotoxic effect of co-treatment approach than rolipram alone, co-treatment did not represent any additive effect on the reduction of phospho-AKT (ser473) and PDE4A levels in comparison with rolipram alone. However, concurrent application of bevacizumab and rolipram not only begot a repressive effect on PDE4A and phospho-AKT (ser473) expressions relative to bevacizumab alone but also rendered an augmentative effect on blockage of the $\mathrm{VEGF}_{\mathrm{A}}$ autocrine loop, upregulation of $\mathrm{p} 53$ and cleaved-caspase 3 levels consistent with a more potent cytotoxicity induction compared to each treatment alone. Alltold, it suggests that GBM tumor propagation may be protected by cancer stem cells via elevating the efflux of $\mathrm{VEGF}_{\mathrm{A}}$ in an autocrine manner that can be effectively controlled by our suggested co-treatment strategy. Finally, current assay highlighted that PDE4, AKT and ERKs pathways can be potential molecular therapeutic targets as an addendum to bevacizumab therapy to impressively restrict the GBM tumor growth.

\section{Conflict of interest}

The authors declare that they do not have any conflict of interests.

\section{Acknowledgments}

We offer our special thanks to Dr. Hassanreza Mohammadi, Dept of Neurosurgery, Shahidbeheshti University of Medical Sciences and all patients, who supported us in sampling procedure. This study was financially supported by Iran University of Medical Sciences (Grant No. 24164) and Iran NATIONAL Science Foundation, Presidency of Islamic Republic of Iran, Vice- presidency for Science and Technology (Grant No. 92035151).

\section{References}

1. Wen PY, Kesari S (2008) Malignant gliomas in adults. New England Journal of Medicine 359 (5):492507

2. Grossman SA, Ye X, Piantadosi S, Desideri S, Nabors LB, Rosenfeld M, Fisher J, Consortium NC (2010) Survival of patients with newly diagnosed glioblastoma treated with radiation and temozolomide in research studies in the United States. Clinical Cancer Research 16 (8):2443-2449

3. Stupp R, Hegi ME, Mason WP, van den Bent MJ, Taphoorn MJ, Janzer RC, Ludwin SK, Allgeier A, Fisher B, Belanger K (2009) Effects of radiotherapy with concomitant and adjuvant temozolomide versus radiotherapy alone on survival in glioblastoma in a randomised phase III study: 5-year analysis of the EORTC-NCIC trial. The lancet oncology 10 (5):459-466 
4. Galli R, Binda E, Orfanelli U, Cipelletti B, Gritti A, De Vitis S, Fiocco R, Foroni C, Dimeco F, Vescovi A (2004) Isolation and characterization of tumorigenic, stem-like neural precursors from human glioblastoma. Cancer research 64 (19):7011-7021

5. Kelly PN, Dakic A, Adams JM, Nutt SL, Strasser A (2007) Tumor growth need not be driven by rare cancer stem cells. Science 317 (5836):337-337

6. Singh SK, Hawkins C, Clarke ID, Squire JA, Bayani J, Hide T, Henkelman RM, Cusimano MD, Dirks PB (2004) Identification of human brain tumour initiating cells. nature 432 (7015):396-401

7. Bao S, Wu Q, McLendon RE, Hao Y, Shi Q, Hjelmeland AB, Dewhirst MW, Bigner DD, Rich JN (2006) Glioma stem cells promote radioresistance by preferential activation of the DNA damage response. nature 444 (7120):756-760

8. Liu G, Yuan X, Zeng Z, Tunici P, Ng H, Abdulkadir IR, Lu L, Irvin D, Black KL, John SY (2006) Analysis of gene expression and chemoresistance of $\mathrm{CD} 133+$ cancer stem cells in glioblastoma. Molecular cancer 5 (1):1

9. Hadjipanayis CG, Van Meir EG (2009) Tumor initiating cells in malignant gliomas: biology and implications for therapy. Journal of molecular medicine 87 (4):363-374

10. Son MJ, Woolard K, Nam D-H, Lee J, Fine HA (2009) SSEA-1 is an enrichment marker for tumorinitiating cells in human glioblastoma. Cell stem cell 4 (5):440-452

11. Singh SK, Clarke ID, Terasaki M, Bonn VE, Hawkins C, Squire J, Dirks PB (2003) Identification of a cancer stem cell in human brain tumors. Cancer research 63 (18):5821-5828

12. Chen W, He D, Li Z, Zhang X, Pan D, Chen G (2015) Overexpression of vascular endothelial growth factor indicates poor outcomes of glioma: a systematic review and meta-analysis. International journal of clinical and experimental medicine $8(6): 8709$

13. Goldhoff P, Warrington NM, Limbrick DD, Hope A, Woerner BM, Jackson E, Perry A, Piwnica-Worms D, Rubin JB (2008) Targeted inhibition of cyclic AMP phosphodiesterase-4 promotes brain tumor regression. Clinical Cancer Research 14 (23):7717-7725

14. Claesson-Welsh L, Welsh M (2013) VEGFA and tumour angiogenesis. Journal of internal medicine 273 (2):114-127

15. Koch S, Tugues S, Li X, Gualandi L, Claesson-Welsh L (2011) Signal transduction by vascular endothelial growth factor receptors. Biochemical Journal 437 (2):169-183

16. Shibuya M (2011) Vascular endothelial growth factor (VEGF) and its receptor (VEGFR) signaling in angiogenesis a crucial target for anti-and pro-angiogenic therapies. Genes \& cancer 2 (12):1097-1105

17. Bao S, Wu Q, Sathornsumetee S, Hao Y, Li Z, Hjelmeland AB, Shi Q, McLendon RE, Bigner DD, Rich JN (2006) Stem cell-like glioma cells promote tumor angiogenesis through vascular endothelial growth factor. Cancer research 66 (16):7843-7848

18. Folkins C, Shaked Y, Man S, Tang T, Lee CR, Zhu Z, Hoffman RM, Kerbel RS (2009) Glioma tumor stemlike cells promote tumor angiogenesis and vasculogenesis via vascular endothelial growth factor and stromal-derived factor 1. Cancer research 69 (18):7243-7251

19. Hamerlik P, Lathia JD, Rasmussen R, Wu Q, Bartkova J, Lee M, Moudry P, Bartek J, Fischer W, Lukas J (2012) Autocrine VEGF-VEGFR2-Neuropilin-1 signaling promotes glioma stem-like cell viability and tumor growth. The Journal of experimental medicine 209 (3):507-520

20. Knizetova P, Ehrmann J, Hlobilkova A, Vancova I, Kalita O, Kolar Z, Bartek J (2008) Autocrine regulation of glioblastoma cell-cycle progression, viability and radioresistance through the VEGF-VEGFR2 (KDR) interplay. Cell cycle 7 (16):2553-2561

21. Xu C, Wu X, Zhu J (2013) VEGF promotes proliferation of human glioblastoma multiforme stem-like cells through VEGF receptor 2. The Scientific World Journal 2013

22. Pullamsetti S, Banat G, Schmall A, Szibor M, Pomagruk D, Hänze J, Kolosionek E, Wilhelm J, Braun T, Grimminger $F$ (2013) Phosphodiesterase-4 promotes proliferation and angiogenesis of lung cancer by crosstalk with HIF. Oncogene 32 (9):1121-1134 
23. Cohen MH, Shen YL, Keegan P, Pazdur R (2009) FDA drug approval summary: bevacizumab (Avastin ${ }^{\circledR}$ ) as treatment of recurrent glioblastoma multiforme. The oncologist 14 (11):1131-1138

24. Hein M, Graver S (2013) Tumor cell response to bevacizumab single agent therapy in vitro. Cancer cell international $13(1): 1$

25. Simon T, Coquerel B, Petit A, Kassim Y, Demange E, Le Cerf D, Perrot V, Vannier J-P (2014) Direct effect of bevacizumab on glioblastoma cell lines in vitro. Neuromolecular medicine 16 (4):752-771

26. Wang L-L, Hu R-C, Dai A-G, Tan S-X (2015) Bevacizumab induces A549 cell apoptosis through the mechanism of endoplasmic reticulum stress in vitro. International journal of clinical and experimental pathology 8 (5):5291

27. Wu H-H, Zhang S, Bian H, Li X-X, Wang L, Pu Y-F, Wang Y-X, Guo C-B (2014) Bevacizumab Regulates Cancer Cell Migration by Activation of STAT3. Asian Pacific journal of cancer prevention: APJCP 16 (15):6501-6506

28. de Groot JF, Fuller G, Kumar AJ, Piao Y, Eterovic K, Ji Y, Conrad CA (2010) Tumor invasion after treatment of glioblastoma with bevacizumab: radiographic and pathologic correlation in humans and mice. Neuro-oncology 12 (3):233-242

29. Moon E-Y, Lee G-H, Lee M-S, Kim H-M, Lee J-W (2012) Phosphodiesterase inhibitors control A172 human glioblastoma cell death through cAMP-mediated activation of protein kinase $A$ and Epac1/Rap1 pathways. Life sciences 90 (9):373-380

30. Smith PG, Wang F, Wilkinson KN, Savage KJ, Klein U, Neuberg DS, Bollag G, Shipp MA, Aguiar RC (2005) The phosphodiesterase PDE4B limits cAMP-associated PI3K/AKT-dependent apoptosis in diffuse large B-cell lymphoma. Blood 105 (1):308-316

31. Tsunoda T, Ota T, Fujimoto T, Doi K, Tanaka Y, Yoshida Y, Ogawa M, Matsuzaki H, Hamabashiri M, Tyson DR (2012) Inhibition of Phosphodiesterase-4 (PDE4) activity triggers luminal apoptosis and AKT dephosphorylation in a 3-D colonic-crypt model. Molecular cancer 11 (1):1

32. von Baumgarten L, Brucker D, Tirniceru A, Kienast Y, Grau S, Burgold S, Herms J, Winkler F (2011) Bevacizumab has differential and dose-dependent effects on glioma blood vessels and tumor cells. Clinical Cancer Research 17 (19):6192-6205

33. Dirix L, Van Dam P, Prove A, Vermeulen P (2010) Bevacizumab in the treatment of patients with advanced breast cancer: where have we landed? Therapeutic advances in medical oncology 2 (5):331342

34. Caporali S, Levati L, Graziani G, Muzi A, Atzori MG, Bonmassar E, Palmieri G, Ascierto PA, D’Atri S (2012) NF-KB is activated in response to temozolomide in an AKT-dependent manner and confers protection against the growth suppressive effect of the drug. Journal of translational medicine 10 (1):1

35. Engelman JA (2009) Targeting PI3K signalling in cancer: opportunities, challenges and limitations. Nature Reviews Cancer 9 (8):550-562

36. Pfaffl MW, Horgan GW, Dempfle L (2002) Relative expression software tool (RESTC) for group-wise comparison and statistical analysis of relative expression results in real-time PCR. Nucleic acids research 30 (9):e36-e36

37. Zuo S-S, Lundahl P (2000) A micro-Bradford membrane protein assay. Analytical biochemistry 284 (1):162-164

38. Jiang BH, Liu LZ (2009) PI3K/PTEN signaling in angiogenesis and tumorigenesis. Advances in cancer research 102:19-65

39. Brautigan DL, Pinault FM (1991) Activation of membrane protein-tyrosine phosphatase involving cAMP-and $\mathrm{Ca} 2+/$ phospholipid-dependent protein kinases. Proceedings of the National Academy of Sciences 88 (15):6696-6700

40. Hong K, Lou L, Gupta S, Ribeiro-Neto F, Altschuler DL (2008) A novel Epac-Rap-PP2A signaling module controls cAMP-dependent Akt regulation. Journal of Biological Chemistry 283 (34):23129-23138 
41. Kang T-W, Choi SW, Yang S-R, Shin T-H, Kim H-S, Yu K-R, Hong I-S, Ro S, Cho JM, Kang K-S (2014) Growth arrest and forced differentiation of human primary glioblastoma multiforme by a novel small molecule. Scientific reports 4

42. Ogawa R, Streiff MB, Bugayenko A, Kato GJ (2002) Inhibition of PDE4 phosphodiesterase activity induces growth suppression, apoptosis, glucocorticoid sensitivity, p53, and p21WAF1/CIP1 proteins in human acute lymphoblastic leukemia cells. Blood 99 (9):3390-3397

43. Arcaro A, Guerreiro AS (2007) The phosphoinositide 3-kinase pathway in human cancer: genetic alterations and therapeutic implications. Current genomics 8 (5):271-306

44. Malmlöf M, Roudier E, Högberg J, Stenius U (2007) MEK-ERK-mediated phosphorylation of Mdm2 at Ser-166 in hepatocytes Mdm2 is activated in response to inhibited Akt signaling. Journal of Biological Chemistry $282(4): 2288-2296$

45. Fulda S, Debatin K (2006) Extrinsic versus intrinsic apoptosis pathways in anticancer chemotherapy. Oncogene 25 (34):4798-4811

46. Haupt S, Berger M, Goldberg Z, Haupt Y (2003) Apoptosis-the p53 network. Journal of cell science 116 (20):4077-4085

47. Bisgin A, Kargi A, Yalcin AD, Aydin C, Ekinci D, Savas B, Sanlioglu S (2012) Increased serum sTRAIL levels were correlated with survival in bevacizumab-treated metastatic colon cancer. BMC cancer 12 (1):1

48. Kranz K, Warnecke A, Lenarz T, Durisin M, Scheper V (2014) Phosphodiesterase type 4 inhibitor rolipram improves survival of spiral ganglion neurons in vitro. PloS one 9 (3):e92157

49. Meyers JA, Taverna J, Chaves J, Makkinje A, Lerner A (2007) Phosphodiesterase 4 inhibitors augment levels of glucocorticoid receptor in B cell chronic lymphocytic leukemia but not in normal circulating hematopoietic cells. Clinical Cancer Research 13 (16):4920-4927

50. Ueno S, Pease ME, Wersinger DMB, Masuda T, Vinores SA, Licht T, Zack DJ, Quigley $H$, Keshet E, Campochiaro PA (2008) Prolonged blockade of VEGF family members does not cause identifiable damage to retinal neurons or vessels. Journal of cellular physiology 217 (1):13-22 
Table1. Characteristics of patients and their tumors

\begin{tabular}{|c|c|c|c|c|c|}
\hline Patients & Gender & Age & Pathological & Tumor location & Purity of GCSCs \\
\cline { 4 - 5 } & & subtype & & CD133 $/$ CD $15^{+}$ \\
\hline 1 & Male & 62 & Primary GBM & Frontal & $27.63 \%$ \\
2 & Female & 60 & Primary GBM & parietal & $96.89 \%$ \\
3 & Male & 51 & Secondary GBM & Frontal & $14.82 \%$ \\
\hline
\end{tabular}



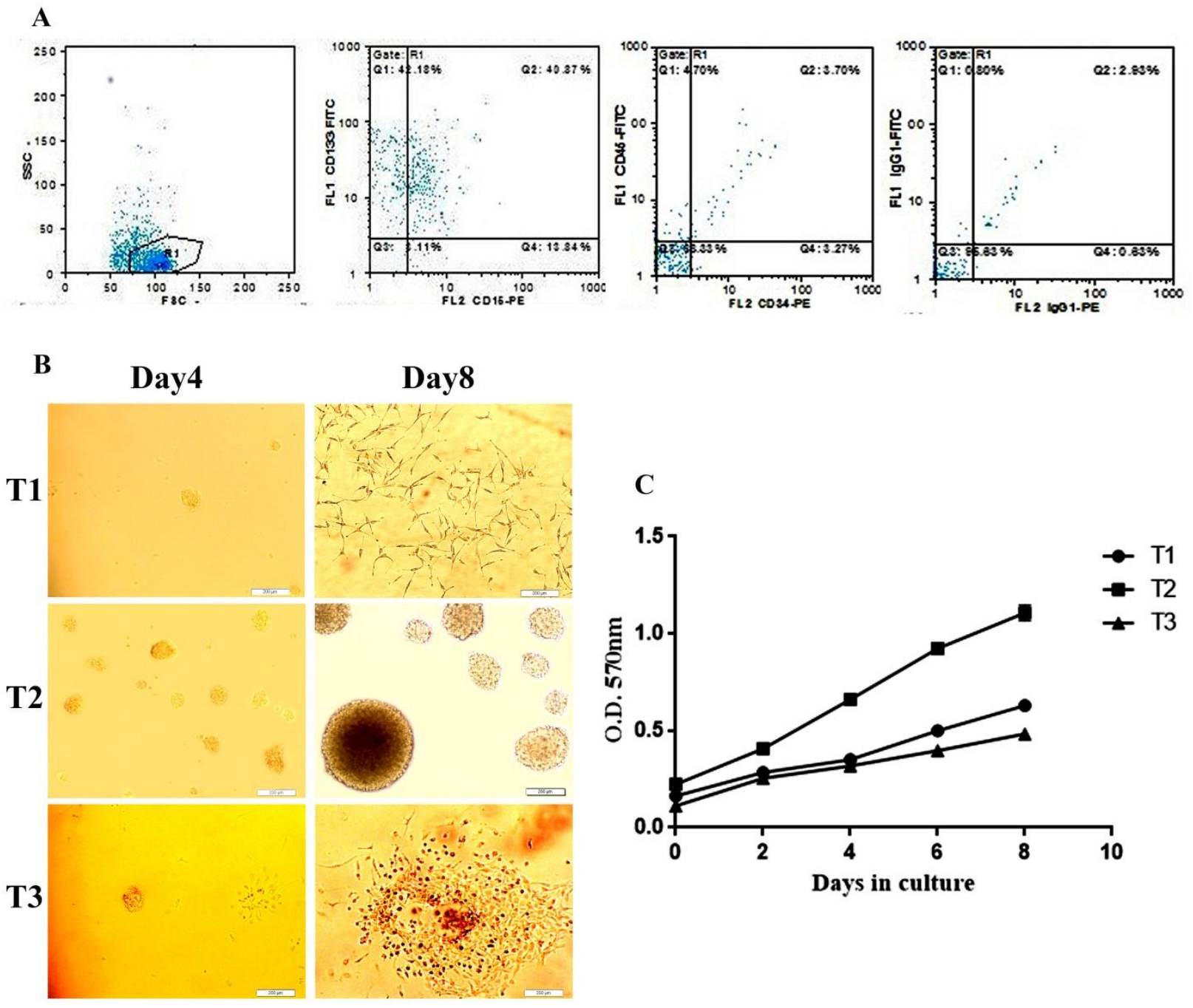

Fig. 1. The results of Flow cytometry for T2-derived spheres on day 8 after plating (A), the ability of colony forming (B) and cell proliferation (C) in all samples over time are depicted. (C) Cell growth curve illustrates that the number of viable T2-derived cells enhanced overtime faster than other samples. The cells at a density of $1 \times 10^{3}$ cells/well were plated in 96 -well plates containing DMEM-F12 supplemented with growth factors, the viable cells quantified by MTT assay through reading absorption spectrums at $570 \mathrm{~nm}$ on a microplate reader. Data are represented as mean $\pm \operatorname{SEM}(n=4)$. 
A

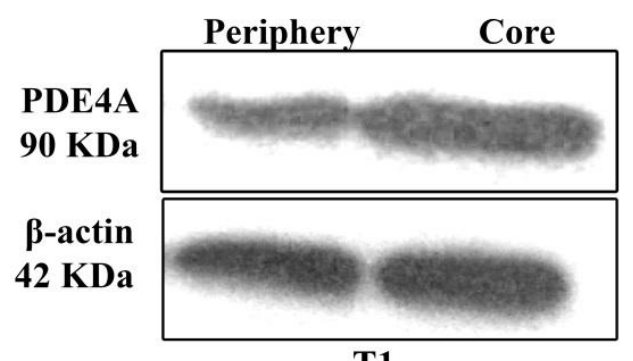

T1

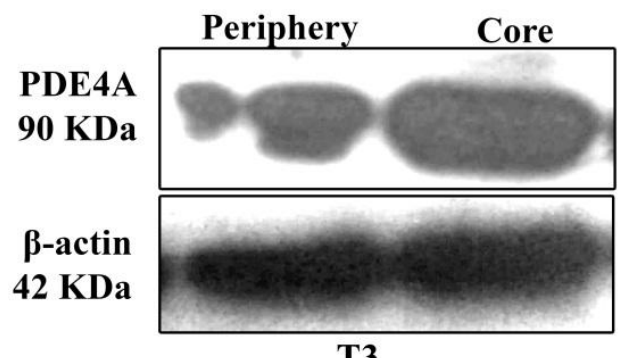

T3

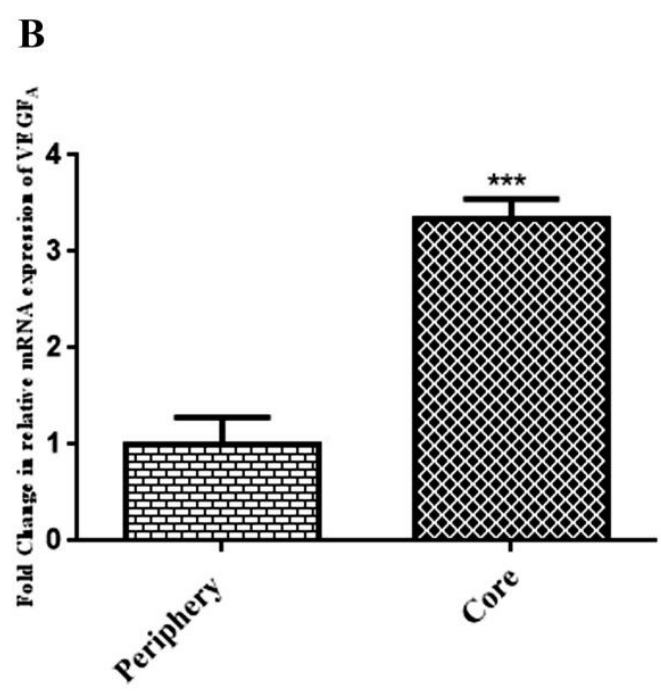

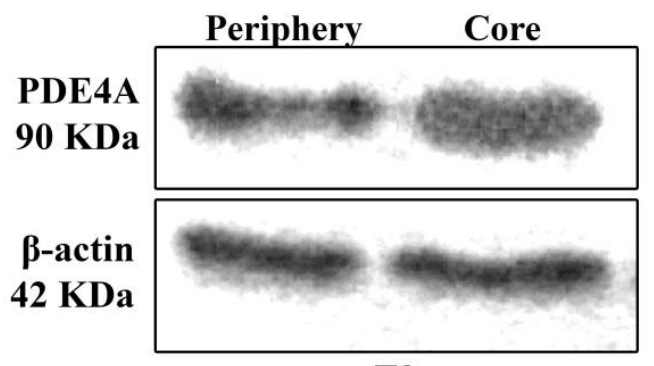

T2

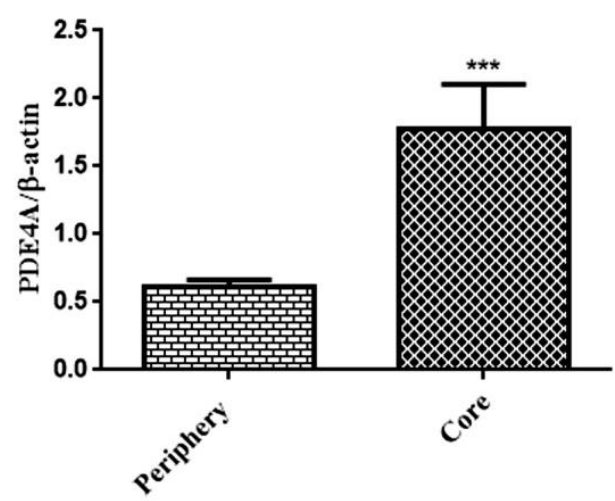

C

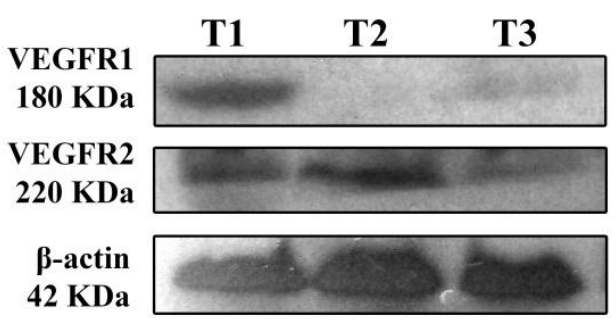

Fig. 2. The PDE4A immunoreactive bands (A), relative values of $V_{E G F}$ mRNA (B) in core and periphery of tumor mass and VEGFR1 and VEGFR2 immunoreactive bands in core-isolated cells are shown. (A) and (B) Tissue samples from three newly diagnosed GBM patients were obtained and lysed to detect regional expression of PDE4A protein and $\mathrm{VEGF}_{\mathrm{A}}$ transcript of each tumor specimen using western blot and qPCR, respectively. Bars represent fold differences of mean normalized expression values \pm SEM $(n=3)$. $* * * P<0.001$ from the respective peripheral zone. (C) Core-isolated cells of all tumor samples were cultured overnight and then collected. Positive VEGFR2 but not VEGFR1 immunoreactivity was represented for all samples by western blot. Beta-actin was examined as housekeeping. 


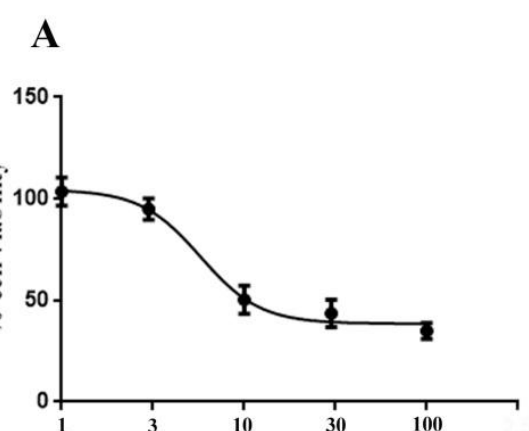

concenteration of bevacizumab $(\mu \mathrm{g} / \mathrm{ml})$

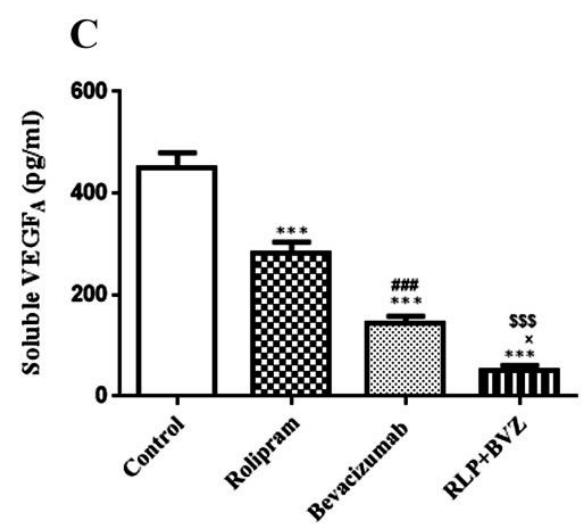

B
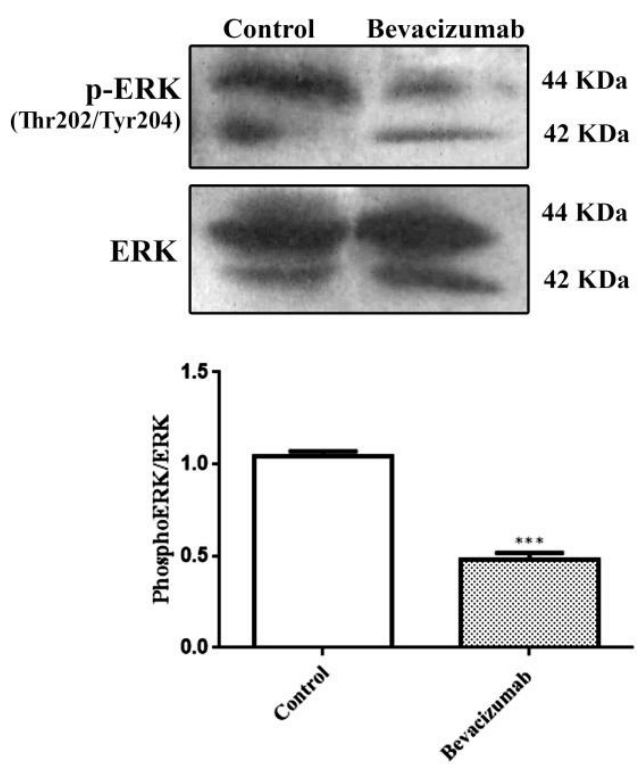

Fig. 3. The effect of bevacizumab (BVZ) alone on the cell viability rate (A), phosphorylation levels of ERKs (B), and free-soluble $\mathrm{VEGF}_{\mathrm{A}}$ levels in the culture supernatant $(\mathrm{C})$ of T2-derived GCSCs as compared to those treated with rolipram (RLP) alone or BVZ+RLP (C) and control (A-C) are illustrated. (A) Non-linear regression curve to calculate $\mathrm{IC}_{50}$ of $\mathrm{BVZ}$ in log scale was plotted by Prism graph pad 6. The cells were incubated with BVZ at various concentrations for $48 \mathrm{~h}$. Cell viability rate was measured using MTT assay by normalizing optical density based on the control. The cells were cultured in presence $\mathrm{BVZ}$ at $\mathrm{IC}_{50}$ or human $\mathrm{IgG1}$ as untreated control (B and C) or RLP $(103 \mu \mathrm{M})$ or BVZ plus RLP (C) for $48 \mathrm{~h}$. (B) Cell lysates were prepared and phospho-ERKs (Thr202/Tyr204) and ERKs were detected using western blot technique. Density of phospho-ERKs was quantified by total lab software and normalized to ERKs density value. (C) Supernatants were collected. Free $\mathrm{VEGF}_{\mathrm{A}}$ levels were quantified by ELISA assay. Data were the representative of four experiments. The comparative significance level was symbolically indicated upper each bar. ${ }^{* * *} P<0.001$ compared to control; \#\#\# $P<0.001$ compared to rolipram; $\times P<0.05$ compared to bevacizumab; $\$ \$ \$ P<0.001$ compared to rolipram 
A

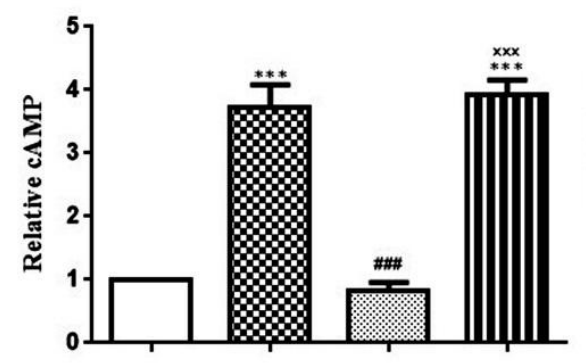

C

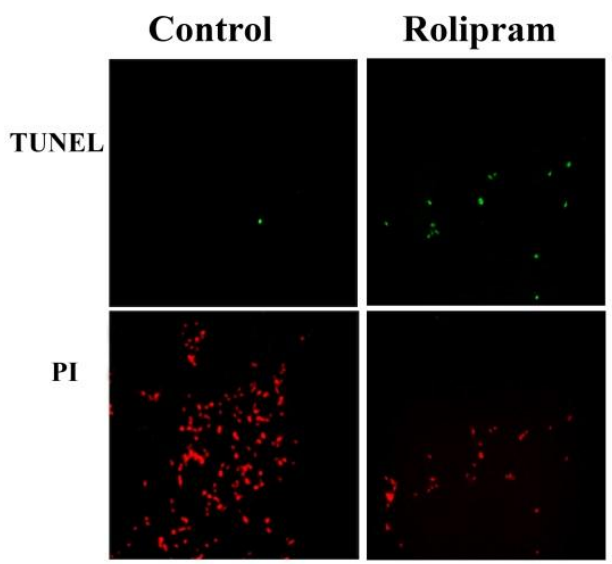

B

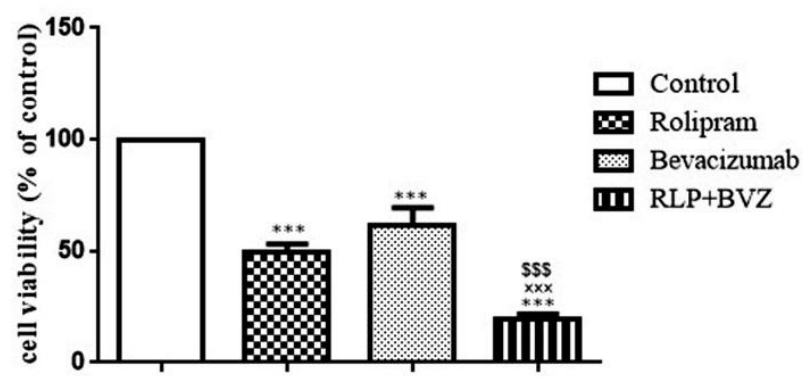

Bevacizuma

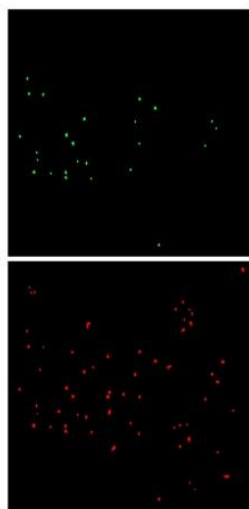

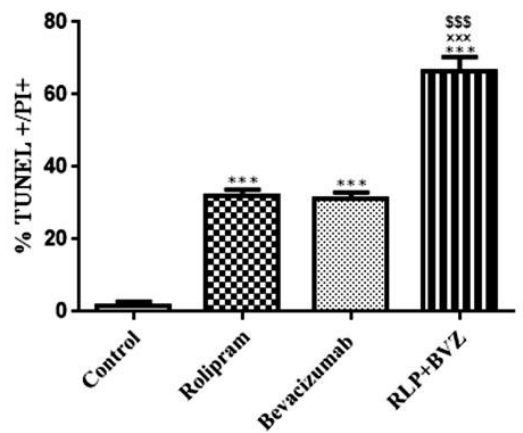

Fig. 4. The effect of bevacizumab (BVZ; $6.5 \mu \mathrm{g} / \mathrm{ml})$ and rolipram (RLP; $103 \mu \mathrm{M})$ either alone or together on the intracellular cAMP levels (A), viability (B) and apoptosis (C) rates of GCSCs isolated from T2 are delineated. The cells incubated with drugs alone or together or human IgG1 as untreated control for $48 \mathrm{~h}$. Intracellular levels of cAMP, the percent of viable and apoptotic cells were measured by Cyclic AMP Enzyme Immunoassay, MTT assay, and TUNEL staining, respectively. Cyclic AMP and cell viability data in each experiment were normalized to control values. Data were the representative of four experiments. The significance level found by post hoc test of one-way ANOVA was symbolically indicated upper each bar. *** $P<0.001$ compared to control; \#\#\# $P<0.001$ compared to RLP; $\times \times \times \quad P<0.001$ compared to $\mathrm{BVZ} ; \$ \$ \$ P<0.001$ compared to RLP. 


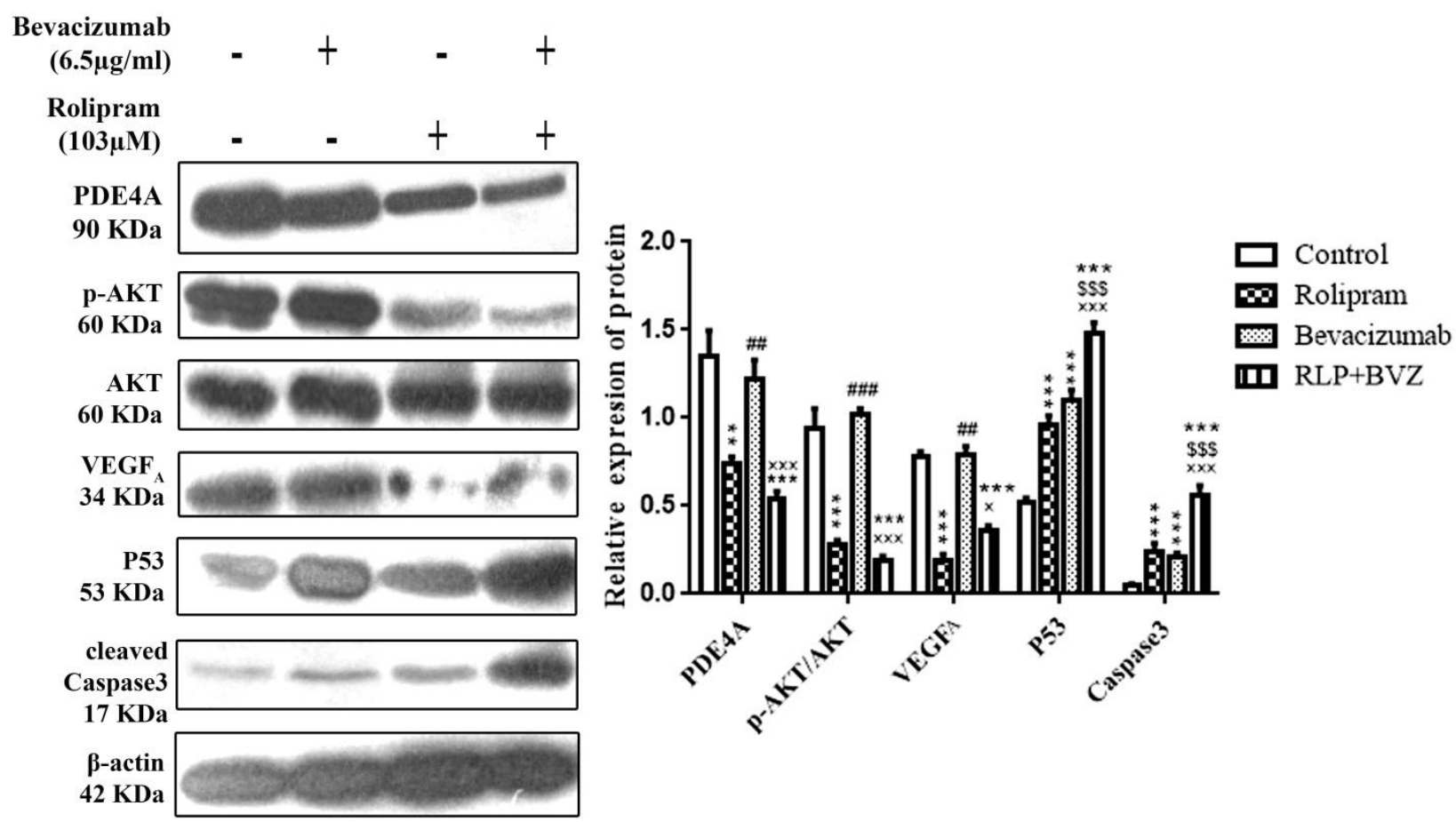

Fig. 5. The effect of bevacizumab (BVZ) and rolipram (RLP) either alone or together on the levels of indicated proteins are portrayed. At $48 \mathrm{~h}$ after treatment, the cells were collected, centrifuged at 4000rpm for $5 \mathrm{~min}$ and then lysed. The endogenous levels of indicated proteins were evaluated using western blot analysis. Semi-quantification of proteins density was accomplished by total lab software and then results were normalized to corresponding density of beta-actin. Data were the representative of three experiments. The significance level found by post hoc test of one-way ANOVA was symbolically indicated upper each bar. $* * * P<0.001$, *** $P<0.01$ compared to control; $\times \times \times P<0.001, \times P<0.05$ compared to bevacizumab; $\$ \$ P<0.001$ and \#\#\# $P<0.001$, \#\# $P<0.01$ compared to rolipram alone. 